\title{
THERMODYNAMIC MODELING OF A PULSE TUBE REFRIGERATION SYSTEM
}

\author{
P. C. Roy ${ }^{1, *}$, B. Kundu ${ }^{1}$
}

\begin{abstract}
Thermodynamic model of the pulse tube refrigeration (PTR) system has been developed based on the ideal gas behaviour to study the cooling effect at the cold end of the refrigerator. Compression and expansion processes of the gas column have been assumed to be isothermal. Mass flow in the regenerator has been evaluated through Ergun's law. Mass flow through the orifice and double inlet valve has been assumed a nozzle flow with a correction factor. Model predicted results have been validated with in-house experimental results as qualitative basis. Model predicted results in compression and expansion processes are also validated with that of the experimental data. Model predicted results are presented to understand the basic phenomenon for the refrigeration effect in various pulse tube refrigerators (BPTR, OPTR and DIPTR). Time duration for expansion process is more than the compression process in case of OPTR and DIPTR, which leads to lower pressure during the expansion and more cooling capacity obtained compared to the BPTR. A distinct comparison among three types of PTRs has been done based on the work done at the cold end. It has been clearly observed that a DIPTR shows better cooling capacity compared to OPTR or BPTR.
\end{abstract}

\section{Keywords: Pulse Tube Refrigeration, Thermodynamic Model, Cooling Effect, Comparison}

\section{INTRODUCTION}

Pulse tube refrigerator (PTR) has a great leverage over other cryo-coolers like Stirling and GM refrigerators due to the absence of moving components at the low temperature end. It has greater reliability due to no sources of mechanical vibration and no clearance seal between piston and cylinder. Moreover, it is a relatively low cost device with a simple and compact design. Though the invention of the pulse tube refrigeration had been in early 60's [1], most of the development was done during last three decades [2,3]. Initially its operating principles were not fully understood. The oscillatory flow inside the pulse tube in the refrigerator and the associated thermodynamic processes are complex which is not similar like the other refrigeration systems [4,5]. As the level of understanding grows gradually, modifications and improved designs yield an improved efficiency. It has now become the most efficient cryocooler for a given size. It is also suitable for a wide variety of applications from civilian to government to military and from ground equipments to space systems. The moving displacer in the Stirling and G-M refrigerators is eliminated by a simple tube in the pulse tube refrigerator. The proper gas motion is achieved by the use of an orifice and a reservoir is used to store the gas during a half cycle. The reservoir volume is large enough that negligible pressure oscillation occurs during the oscillating flow. The oscillating flow through the orifice separates the heating and cooling effects just as the displacer does for the Stirling and G-M refrigerators. The orifice pulse tube refrigerator (OPTR) operates ideally with adiabatic compression and expansion. The function of the pulse tube is to insulate the processes at its two ends. Gas in the middle portion of the pulse tube never leaves the pulse tube and thus forming a temperature gradient that insulates the two ends from each other [6]. The pulse tube is the unique component in this refrigerator. Though it is an open tube, the thermo-hydrodynamic processes involved are extremely complex and not well understood or successfully modelled [6]. Its function is to transmit hydrodynamic power in an oscillating gas system from one end to the other end across a temperature gradient with a minimum of power dissipation and entropy generation. The basic pulse-tube refrigerator (BPTR) consists of a pressure-wave generator, a regenerator, and a tube only. There are no orifices of any kind. The operation is based on the thermal interaction between the gas and the tube wall. Performance of the BPTR is improved by adding an orifice and a buffer commonly known as Orifice pulse tube refrigerator (OPTR), but mass flow through the regenerator increases leading to a degradation of regenerator performance. In the double-inlet PTR (DIPTR), the hot end of the pulse tube is connected with the entrance (hot end) of the regenerator by a valve. The double inlet is a bypass for the regenerator and the tube, and it reduces the dissipation in the regenerator. Many researchers have This paper was recommended for publication in revised form by Sandip Kale

${ }^{1}$ Department of Mechanical Engineering, Jadavpur University, Kolkata, INDIA

${ }^{\star} E$-mail address: prokash.roy@gmail.com

Manuscript Received 14 June 2016, Accepted 11 December 2016 
developed models to predict the performance of the PTRs based on some empirical parameters, which differs from author to author. Thermodynamic analysis of the pulse tube refrigeration system is not new. But for the purpose of analysis, researchers have used different schemes for dividing the full pulse tube refrigerator into subsystems. Zhu et al. [3] proposed to divide the pulse tube section into three subsystems, the middle section of the tube separating the cold and the hot volumes. In their approach, no heat exchange occurs in the mid-section, and that pressure variation induces temperature swing in the gas. Liang et al. [7] developed a model to understand the working mechanism of three common types of pulse tube refrigerator by analyzing the thermodynamic behaviour of gas elements as adiabatic process in the pulse tube. They assumed the pressure variation inside the pulse tube to be symmetric at the time of compression and expansion. For the simplicity of calculations they took it as trapezoidal variation with time. Recently, CFD models are more popular because of its complexity geometry and it takes more computation time compare to thermodynamic models $[8,9,10]$ developed a two-dimensional computational fluid dynamic (CFD) simulation of a GM type double inlet pulse tube refrigerator (DIPTR), operating under a variety of thermal boundary conditions analyzed by using commercial CFD package to model the oscillating flow inside a pulse tube refrigerator to predict the performance. Chen et al. [11] studied the thermodynamic cycles in an instance tube pulse tube refrigerator (ITPTR) by means of CFD techniques. They observed that gas parcels working in different parts of ITPTR undergo different thermodynamic cycles. A one-dimensional finite volume discretization model is proposed implemented in a computer program for the modeling of a Stirling type Pulse Tube Cryocoolers (PTC) to study the performance. Temperature dependency of thermo-physical properties are taken into account between the working fluid and the solid parts, and heat conductions in the gas and solid [12]. A detailed time-dependent axisymmetric computational fluid dynamic (CFD) model of the OPTR has also been developed to predict the performance by Antao and Farouk [13]. An improved representation of heat transfer in the porous media is achieved by employing a thermal non-equilibrium model to couple the gas and solid (porous media) energy equations. Dai et al. [14] developed model to investigate the three dimensional oscillating flow and heat transfer in the pulse tube and heat exchangers of a pulse tube refrigerator. Zhang et al. [9] developed a linear model for theoretical analyses for the orifice and double-inlet PTCs that indicates the cooling performance can be improved by introducing the cold reservoir. Park et al. [15] also analyzed to identify loss mechanisms with the simple numerical computation (linear model) followed by Zhang et al. [16] which considers the dynamic characteristics of the cold linear compressor with thermo-hydraulic governing equations for each of sub components of the PTR. All the mass flows and pressure waves were assumed to be sinusoidal. But practically it never happens.

In the present work, the variation of stroke volume of the compressor cylinder is considered sinusoidal in nature. In this model, the compression and expansion processes are taken as isothermal and thus it is reversible observed by Neveu and Babo [17]. In addition, it shows a higher efficiency than the adiabatic or any other model of the pulse tube. They assumed that each volume is subjected to several complex processes. These are due to heat exchange in the associated heat exchanger, surface heat pumping in the tube and the mixing of the gas. They expressed all these phenomena through lumped parameters, considering isothermal heat exchange. They developed the model only for Stirling type orifice pulse tube refrigerator. In the present work, an isothermal model has been developed that has ability to predict the performance any basic PTRs and a comparison among them has been done.

\section{MODEL DESCRIPTIONS}

A pulse tube refrigeration system is a closed system that uses an oscillating pressure (typically produced by a compressor) at one end to generate an oscillating gas flow in the rest of the system. The schematic arrangement of a Stirling type pulse tube refrigerator (PTR) system is shown in Figure 1.

The pulse tube device is divided into six open subsystems. Three of them exchange work, heat and mass with the surroundings (compressor, cold and hot volumes), while the others exchange mass only (regenerator, double inlet valve and orifice-reservoir). It has been assumed that all heat exchanges occur at a constant temperature, and that temperature of all subsystems exchanging heat are equal to those of the heat reservoirs. Another condition is that mechanical equilibrium is realized in each part of the device. These conditions lead to the model presented in Figure 2. 


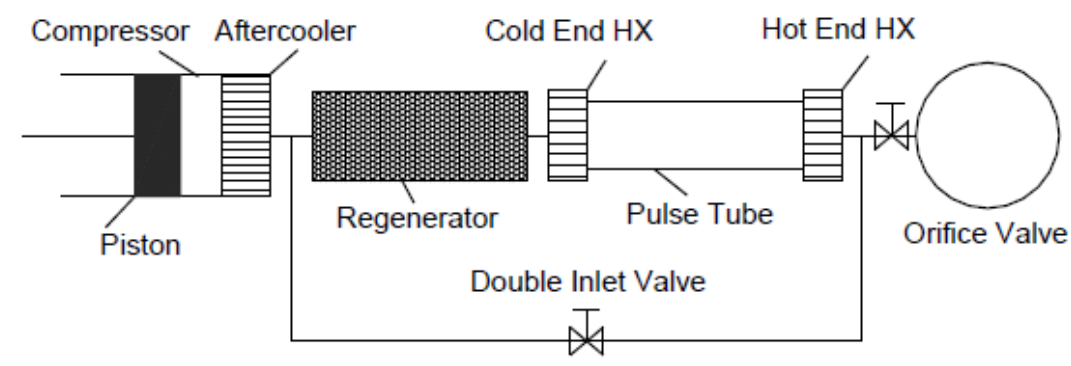

Figure 1. Pulse Tube Refrigerator System

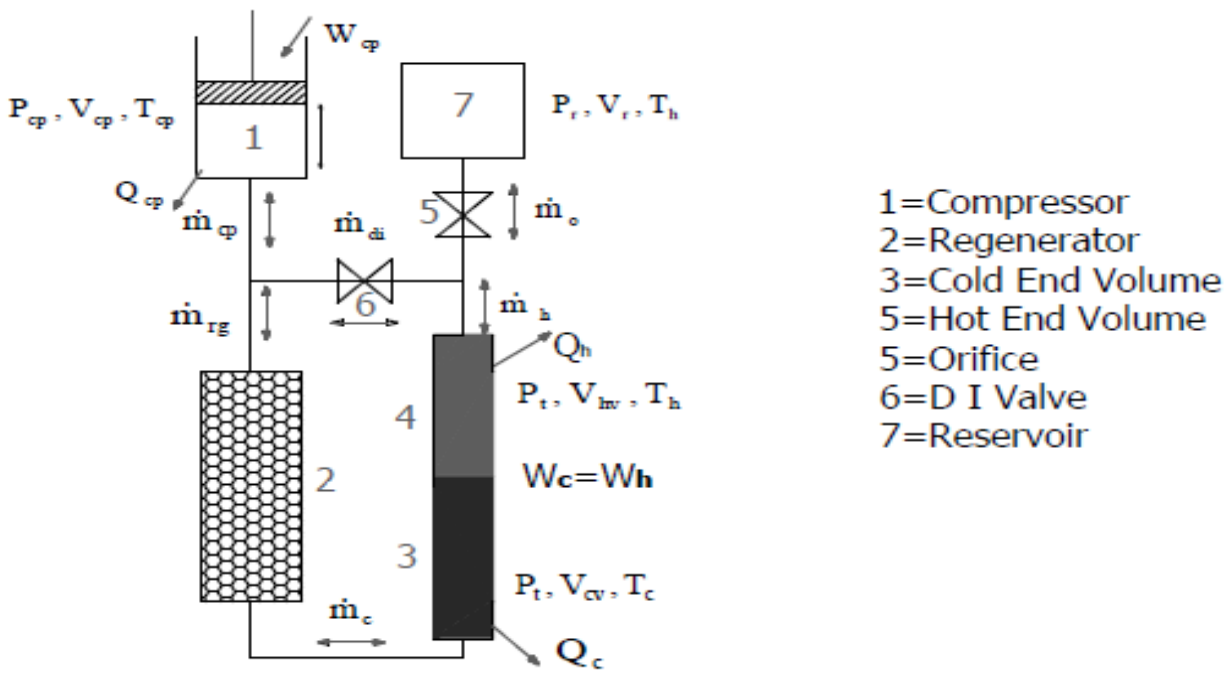

Figure 2. Schematic diagram of the model

The system described in the Figure 2 consists of six opened subsystems.

- Isothermal compressor at $T_{c p}$, exchanging mechanical work $\dot{w}_{c p}$ and heat $\dot{q}_{c p}$ with the surroundings at temperature $T_{h}$ and mass flow rate $\dot{m}_{c p}\left(\dot{m}_{r g}+\dot{m}_{d i}\right)$, where, $\dot{m}_{r g}$ with the regenerator and $\dot{m}_{d i}$ with the double inlet valve.

- Regenerator, exchanging mass rate $\dot{m}_{r g}$ at $T_{c p}$ with the compressor $\dot{m}_{c}$ and at $T_{c}$ with the pulse tube.

- Cold volume in the pulse tube at $T_{c}$, exchanging heat $\dot{q}_{c}$ with the surrounding at temperature $T_{c}$, mass $\dot{m}_{c}$ with the regenerator, and mechanical work $\dot{w}_{c}$ with the hot volume.

- Hot volume in the pulse tube at $T_{h}$, exchanging mechanical work $\dot{w}_{h}$ with the cold volume, heat $\dot{q}_{h}$ with the surrounding at temperature $T_{c p}$ and mass $\dot{m}_{h}\left(\dot{m}_{o}-\dot{m}_{d i}\right)$ with the reservoir via orifice and DI valve.

- Adiabatic orifice and reservoir, exchanging mass $\dot{m}_{o}$ with hot volume.

- Double inlet valve exchanging mass $\dot{m}_{d i}$ with compressor and hot volume.

Each subsystem has two states, viz. gas and wall. Conservation of mass and energy has been applied to the six subsystems with the following assumptions:

- Ideal perfect gas,

- Uniform temperature in each subsystem,

- Mechanical equilibrium in compressor and pulse tube, ideal regenerator, i.e. front wave moving back and forth during a cycle,

- Piston movement in the pulse tube.

- Negligible gas volume in the regenerator compared to compressor, tube and reservoir.

- Negligible pressure losses due to pipe and bends. 


\section{GOVERNING EQUATIONS}

Isothermal opened variable volume:

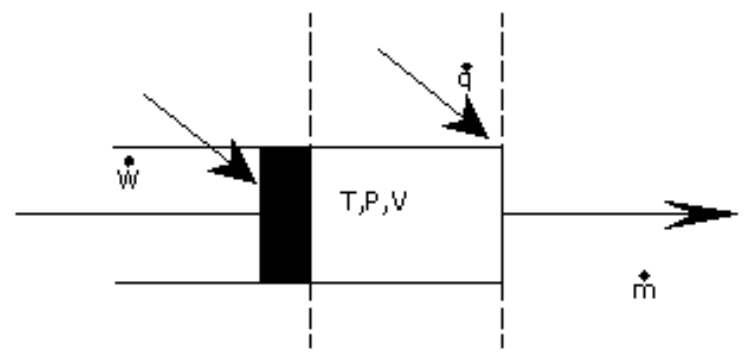

Figure 3. Isothermal volume model

From (Figure 3) mass conservation and First law and thermodynamics [17],

$$
\begin{aligned}
& \frac{d m}{d t}=\dot{m} \\
& \frac{d U}{d t}=\dot{w}+\dot{q}+\dot{m} h
\end{aligned}
$$

Assuming gas in the pulse tube as ideal gas

$$
P V=m R T
$$

Equation (2) can be written as

$$
d m=m\left(\frac{d P}{P}+\frac{d V}{V}-\frac{d T}{T}\right)
$$

Change of the internal energy of the gas inside the system volume can be written as [17],

$$
d U=m c_{v} d T+u d m
$$

As temperature has been assumed constant, $d T=0$, so that Equations ( 4 and 5) can be simplified as

$$
\begin{aligned}
& d m=m\left(\frac{d P}{P}+\frac{d V}{V}\right)=\frac{1}{R T}(V d P+P d V) \\
& d U=u d m
\end{aligned}
$$

Substituting Eq. (6) in Eq. (2), and assuming mechanical equilibrium in the system volume,

$$
\begin{aligned}
& \frac{d P}{d t}=\frac{1}{V}\left(\dot{m} R T-P \frac{d V}{d t}\right), \\
& \dot{q}=P \frac{d V}{d t}-\dot{m}(P V)=P \frac{d V}{d t}-\dot{m} R T, \\
& \dot{w}=-P \frac{d V}{d t}
\end{aligned}
$$

\section{For Isothermal Compressor}

Applying the above sets of equations to the compressor, Eq. (7) becomes 


$$
\begin{aligned}
& \frac{d P_{c p}}{d t}=\frac{1}{V_{c p}}\left(-\dot{m}_{c p} R T_{c p}-P_{c p} \frac{d V_{c p}}{d t}\right), \\
& \dot{q}_{c p}=P_{c p} \frac{d V_{c p}}{d t}+\dot{m}_{c p} R T_{c p}, \\
& \dot{w}_{c p}=-P_{c p} \frac{d V_{c p}}{d t}
\end{aligned}
$$

\section{For Pulse tube}

Similarly to that in compressor, the pulse tube flow has been assumed to be a piston like flow. The pulse tube has been divided into two distinct volumes, one for cold volume, $V_{c}$, and the other for the hot volume $V_{h}$ at uniform temperature to ensure the reversibility of the model.

For cold volume,

and for hot volume,

$$
\begin{aligned}
& \frac{d P_{t}}{d t}=\frac{1}{V_{c}}\left(\dot{m}_{c} R T-P_{t} \frac{d V_{c}}{d t}\right), \\
& \dot{q}_{c}=P_{t} \frac{d V_{c p}}{d t}-\dot{m}_{c} R T_{c}, \\
& \dot{w}_{c}=-P_{t} \frac{d V_{c}}{d t}
\end{aligned}
$$

Mechanical equilibrium yields

$$
\begin{aligned}
& \frac{d P_{t}}{d t}=\frac{1}{V_{h}}\left(-\left(\dot{m}_{o}-\dot{m}_{d i}\right) R T-P_{t} \frac{d V_{h}}{d t}\right), \\
& \dot{q}_{h}=P_{t} \frac{d V_{h}}{d t}+\left(\dot{m}_{o}-\dot{m}_{d i}\right) R T_{h}, \\
& \dot{w}_{h}=P_{t} \frac{d V_{h}}{d t}
\end{aligned}
$$

$$
\dot{w}_{c}+\dot{w}_{h}=0
$$

Combining Eqs. (8) to (10) leads to the following set of equations, describing the pulse tube:

$$
\begin{aligned}
& \frac{d P_{t}}{d t}=\frac{1}{V_{t}}\left(\dot{m}_{c} R T_{c}-\left(\dot{m}_{o}-\dot{m}_{d i}\right) R T_{h}\right), \\
& \frac{d X_{t}}{d t}=\frac{1}{P_{t} V_{t}}\left(\dot{m}_{c} R T_{c}\left(1-X_{t}\right)+\left(\dot{m}_{o}-\dot{m}_{d i}\right) R T_{h} X_{t}\right), \\
& \dot{q}_{c}=P_{t} V_{t} \frac{d X_{t}}{d t}-\dot{m}_{c} R T_{c}, \\
& \dot{q}_{h}=-P_{t} V_{t} \frac{d X_{t}}{d t}+\left(\dot{m}_{o}-\dot{m}_{d i}\right) R T_{h},
\end{aligned}
$$

where $X_{t}=\frac{V_{c}}{V_{t}}=$ the dimensionless cold volume. 


\section{Orifice plus reservoir:}

Applied to the reservoir, Eq. (7) becomes

$$
\begin{aligned}
& \frac{d P_{r}}{d t}=\frac{1}{V_{r}}\left(-\dot{m}_{o} R T_{h}\right) \\
& \dot{q}_{r}=\dot{m}_{o} R T_{h} \\
& \dot{w}=0,
\end{aligned}
$$

Mass flow in the regenerator has been evaluated through Ergun's law. Mass flow through the orifice and double inlet valve has been assumed a nozzle flow with a correction factor [18].

\section{Change in Compressor Volume:}

Sinusoidal variation has been taken for the compressor volume

$$
V_{c p}(t)=V_{0}+\frac{V_{s}}{2}[1+\sin (2 \pi f t)]
$$

\section{SOLVING METHODOLOGY}

It has been assumed that temperatures are constant and uniform at short time scale. Then the whole device can be described by the following four properties: $\mathrm{P}_{\mathrm{cp}}, \mathrm{P}_{\mathrm{t}}, \mathrm{P}_{\mathrm{r}}$ and $\mathrm{X}_{\mathrm{t}}$ varying in time according to the differential equations described above.

$$
\begin{aligned}
& \frac{d P_{c p}}{d t}=\frac{1}{V_{c p}}\left(-\dot{m}_{c p} R T-P_{c p} \frac{d V_{c p}}{d t}\right), \\
& \frac{d P_{t}}{d t}=\frac{1}{V_{t}}\left(\dot{m}_{r g} R T_{c}-\left(\dot{m}_{h}-\dot{m}_{d i}\right) R T_{h}\right), \\
& \frac{d X_{t}}{d t}=\frac{1}{P_{t} V_{t}}\left(\dot{m}_{r g} R T_{c}\left(1-X_{t}\right)+\left(\dot{m}_{o}-\dot{m}_{d i}\right) R T_{h} X_{t}\right), \\
& \frac{d P_{r}}{d t}=\frac{1}{V_{r}}\left(-\dot{m}_{o} R T_{h}\right)
\end{aligned}
$$

These are all time varying ordinary differential equations and need only one initial condition for each to solve. From equation (13), the following initial conditions are derived as shown in figure 4 .

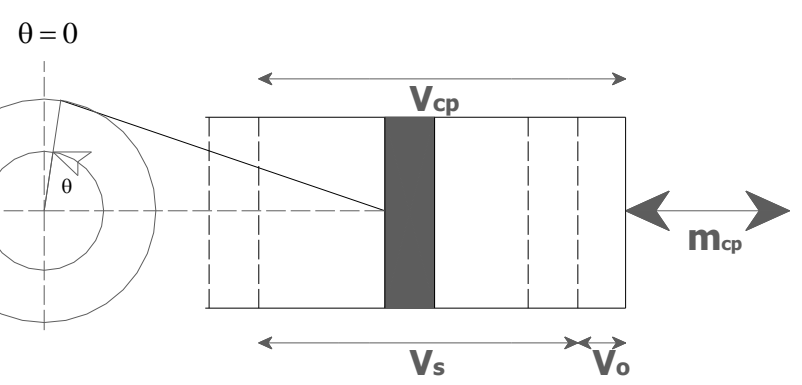

Figure 4. Variation of volume with time or angle

At $t=0$, it can be assumed that $\theta=\omega t=2 \pi f t=0$ 
This means that the piston will be at middle position when the compressor is at average compressor pressure. Initially the whole system is in equilibrium state, the pressure at the pulse tube and the reservoir will be at same pressure. As the flow in the pulse tube has been assumed as piston like flow, the gas piston will be at the middle position. Therefore,

$$
X_{t}(t)=X_{t}(0)=\frac{V_{c}(0)}{V_{t}}=0.25
$$

Fourth order Runge-kutta Method has been used to calculate Pcp, Pt, $\operatorname{Pr}$ and $X t$ with respect to time. Design data of the model are listed in Table 1.

Table 1. Design data for the model

\begin{tabular}{|l|l|}
\hline \multicolumn{1}{|c|}{ Components } & \multicolumn{1}{c|}{ Parameters } \\
\hline Compressor & $\begin{array}{l}\text { Dead volume }=\mathrm{V}_{\mathrm{o}}=0.13 \times 10^{-3} \mathrm{~m}^{3} \\
\text { Swept volume }=\mathrm{V}_{\mathrm{s}}=0.5 \times 10^{-3} \mathrm{~m}^{3}\end{array}$ \\
\hline Regenerator & $\begin{array}{l}\text { Length }=\mathrm{L}_{\mathrm{rg}}=0.3 \mathrm{~m} \\
\text { Diameter }=\mathrm{D}_{\mathrm{rg}}=0.032 \mathrm{~m} \\
\text { Porosity }=0.7 \\
\text { Hydraulic diameter: } 0.04 \mathrm{~mm} .\end{array}$ \\
\hline Pulse tube & $\begin{array}{l}\text { Length }=\mathrm{L}_{\mathrm{t}}=0.8 \mathrm{~m} \\
\text { Diameter }=\mathrm{D}_{\mathrm{t}}=0.02 \mathrm{~m} \\
\end{array}$ \\
\hline Orifice & Volume $=\mathrm{V}_{\mathrm{t}}=0.00025 \mathrm{~m}^{3}$ \\
\hline DI valve & Diameter $=1 \mathrm{~mm}$ \\
\hline Reservoir & Diameter $=1 \mathrm{~mm}$ \\
\hline Average pressure & Volume $=0.007 \mathrm{~m}^{3}$ \\
\hline Frequency & 10 bar \\
\hline Cold end temperature & $2 \mathrm{~Hz}$ \\
\hline Hot end temperature & $100 \mathrm{~K}$ \\
\hline Helium gas at 10 bar and $200 \mathrm{~K}$ temperature & $300 \mathrm{~K}$ \\
& Viscosity $=\mu=15.21 \mathrm{X} 10^{-6} \mathrm{Ns} / \mathrm{m}^{2}$ \\
& Density $=\rho=2.389 \mathrm{Kg} / \mathrm{m}^{3}$ \\
$c_{p}=5193.0 \mathrm{~J} / \mathrm{Kg} \mathrm{K}$ \\
$\mathrm{R}=2074.6 \mathrm{~J} / \mathrm{Kg} \mathrm{K}$ \\
$\mathrm{K}=1.66$ \\
\hline
\end{tabular}

\section{RESULTS AND DISCUSSION}

The present model has predicted the pressure variation in the compressor, pulse tube and reservoir (constant) with time in case of BPTR, OPTR and DIPTR configurations which have been presented as shown in Figure 5-7. In the present study, an effort has been made for a qualitative comparison of pressure variation at different section of the pulse tube between the experimental results and model predicted results. It is noted that developed model is based on Stirling type arrangement and experimental studies are carried out with GM type. Experimental details are given in the reference, Roy [18]. Pressure variation at regenerator inlet, pulse tube and reservoir with respect to time in a GM type pulse tube refrigerator has been shown in Figure 8. In GM type pulse tube refrigerator, a rotary valve is used to switch system pressure with high pressure (HP) and low pressure (LP) lines of the compressor to create compression and expansion process in the pulse tube. It is observed from both the results that although, there have some quantitative difference between experimental and model prediction, a distinct phase relationship among the pressures are observed in both experimental results and model predicted results as shown in Table 2. Pulse tube refrigerator has been operated at a frequency of $2 \mathrm{~Hz}$. Model predicted results of compression and expansion duration (time) are well validated with the experimental data. Variations of maximum and minimum pressure variation in compressor and pulse tube are due to its inherent types of the refrigerator. 
Table 2. Data for model validation

\begin{tabular}{|l|c|c|}
\hline Data Observed & $\begin{array}{l}\text { Experimental data } \\
\text { (GM Type) }\end{array}$ & $\begin{array}{l}\text { Model Prediction } \\
\text { (Stirling Type) }\end{array}$ \\
\hline Compression duration & $0.19 \mathrm{~s}$ & $0.21 \mathrm{~s}$ \\
\hline Expansion duration & $0.31 \mathrm{~s}$ & $0.29 \mathrm{~s}$ \\
\hline
\end{tabular}

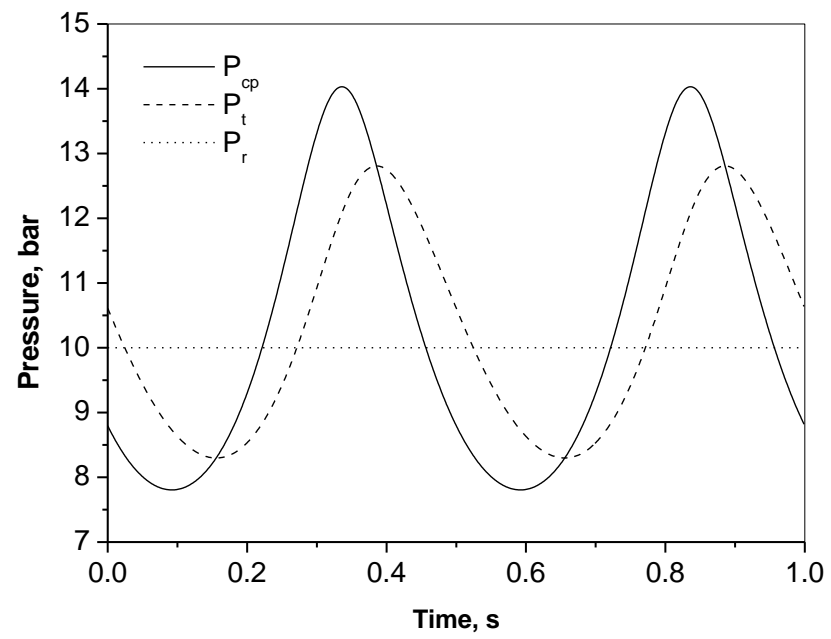

Figure 5. Pressure variation of compressor $\left(\mathrm{P}_{\mathrm{cp}}\right)$, pulse tube $\left(\mathrm{P}_{\mathrm{t}}\right)$ and reservoir $\left(\mathrm{P}_{\mathrm{r}}\right)$ with time in case of BPTR.

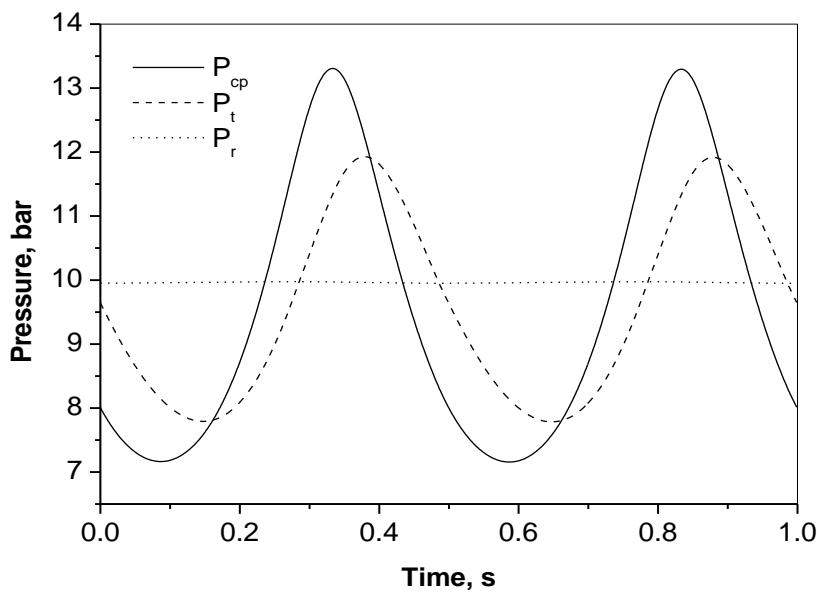

Figure 6. Pressure variation of compressor $\left(\mathrm{P}_{\mathrm{cp}}\right)$, pulse tube $\left(\mathrm{P}_{t}\right)$ and reservoir $\left(\mathrm{P}_{\mathrm{r}}\right)$ with time in case of OPTR

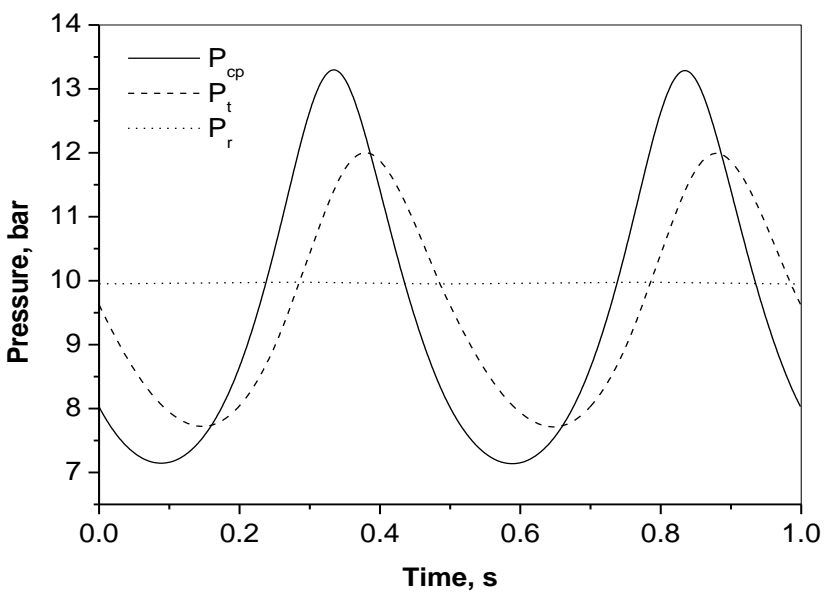

Figure 7. Pressure variation of compressor $\left(\mathrm{P}_{\mathrm{cp}}\right)$, pulse tube $\left(\mathrm{P}_{t}\right)$ and reservoir $\left(\mathrm{P}_{\mathrm{r}}\right)$ with time in case of DIPTR. 


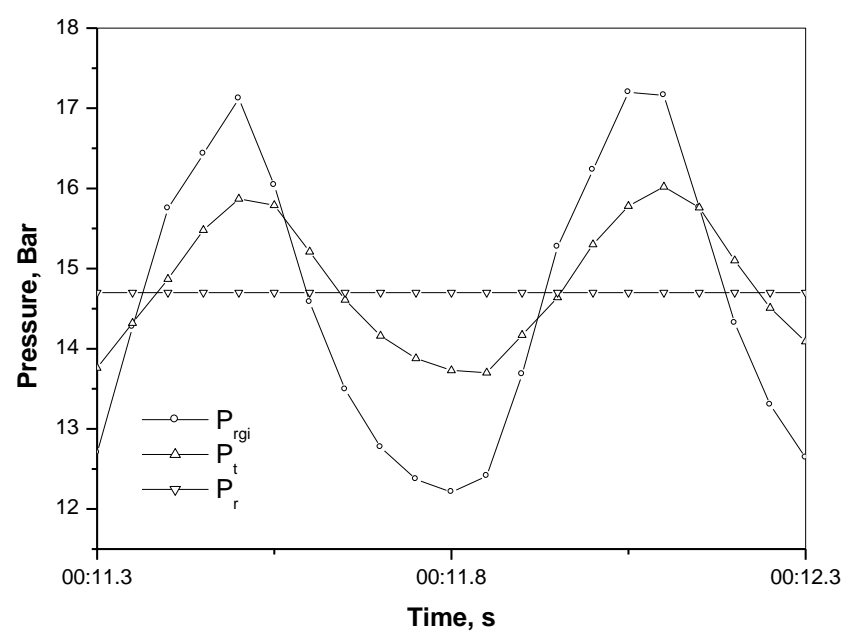

Figure 8. Pressure variation at regenerator inlet, pulse tube and reservoir with respect to time assuming reservoir pressure constant (average pressure).

In Figure 5, pressure variation at the compressor, pulse tube and reservoir are presented in a frequency of $2 \mathrm{~Hz}$. Time duration of compression and expansion are same, 24s, since there is no reservoir, whole masses are compressed and expanded in a closed system. Maximum pressure rise at the compressor and pulse tube are 13.9 bar and 12.6 bar, respectively whereas minimum pressure during expansion are 7.8 bar and 8.4 bar, respectively. The average pressure difference (10bar) in expansion process is less compared to the compression process. It means that BPTR are not capable to reach minimum pressure or temperature at the cold end of the pulse tube. On the other end, in OPTR and DIPTR, time taken for expansion process is more ( $\sim 29 \mathrm{~s})$ compared to compression process $(\sim 0.21 \mathrm{~s})$. It leads to increase more pressure different during expansion process. Minimum pressure at the compressor and pulse tube during expansion are 7.1 bar and 7.7 bar, respectively. So, OPTR and DIPTR are capable to reach minimum temperature compared to that in BPTR.

With this above qualitative validation of the developed model, a comparison among the BPTR, OPTR, and DIPTR have been made based on the cold end work done produced in different types of PTRs. Volume variation of compressor $(\mathrm{CP})$, cold end (CE) and hot end (HE) with time in case of BPTR are presented in Figure 9. Now compressor (CP), cold end (CE) and hot end (HE) work-done in a cyclic operation in case of BPTR has been presented in Figure 10 by combining Figure 5 and 9. It has been observed from Figure 10 that no PV work occurs at the cold end as well as at the hot end volume. It indicates that there is no cooling or heating effect in case of BPTR. This result supports the fact that surface heat pumping mechanism is responsible for cooling in BPTRs. But in case of Figure 11 and 12, a certain amount of PV work done have been observed due to adequate phase relationship between pressure and mass flow rate occurs in OPTR and DIPTR. Similar observations have been made for gas parcels in the pulse tube by Chen et al. [11] and Qui et al. [19].

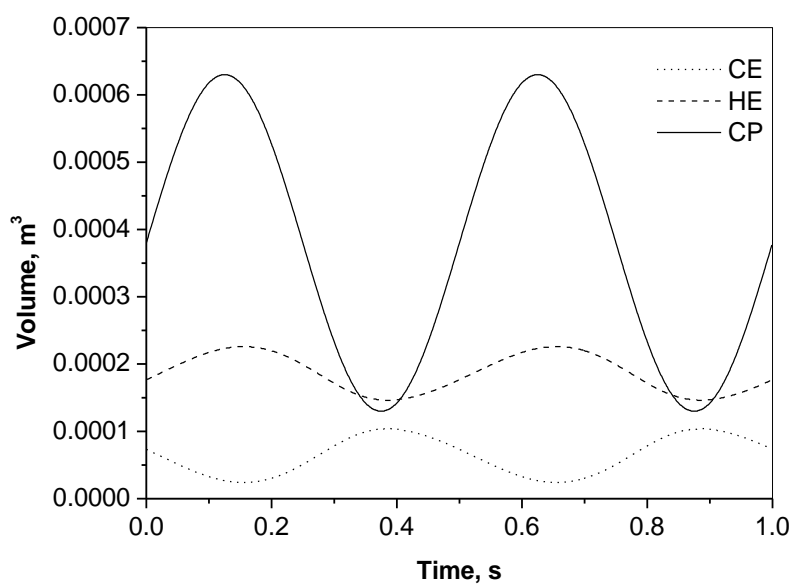

Figure 9. Volume variation of compressor (CP), cold end (CE) and hot end (HE) with time in case of BPTR. 


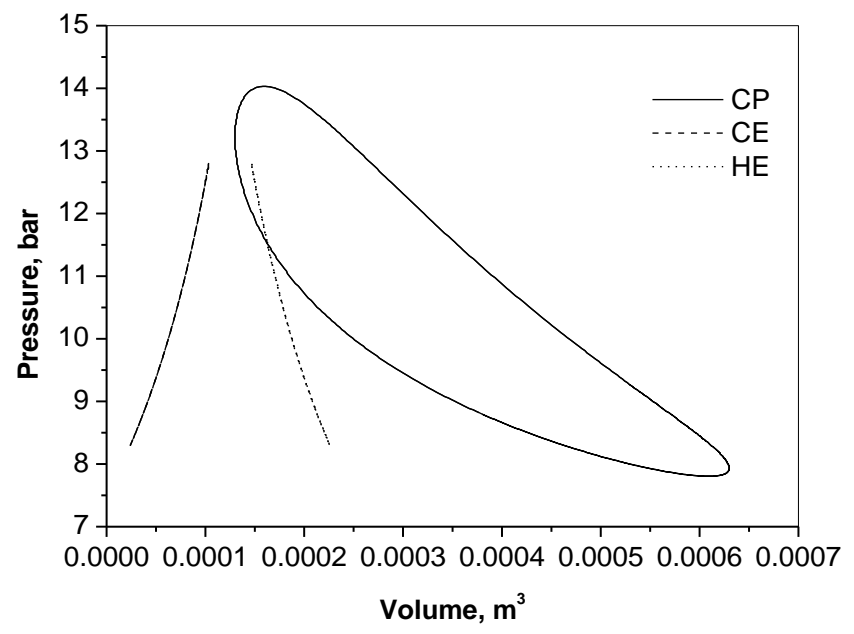

Figure 10. Compressor (CP), cold end (CE) and hot end (HE) work done in a cyclic operation in case of BPTRs.

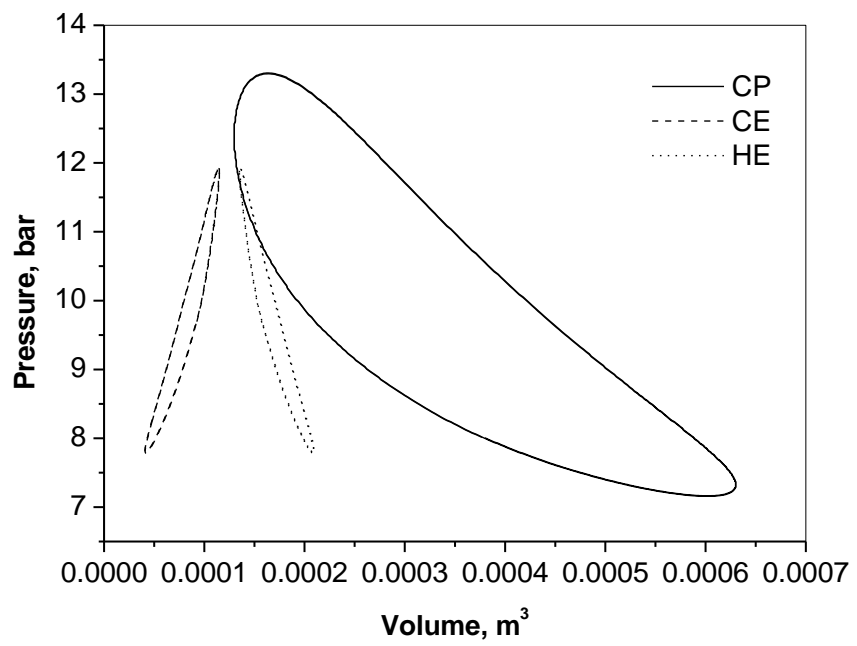

Figure 11. Compressor (CP), cold end (CE) and hot end (HE) work done in cyclic operation in case of OPTRs

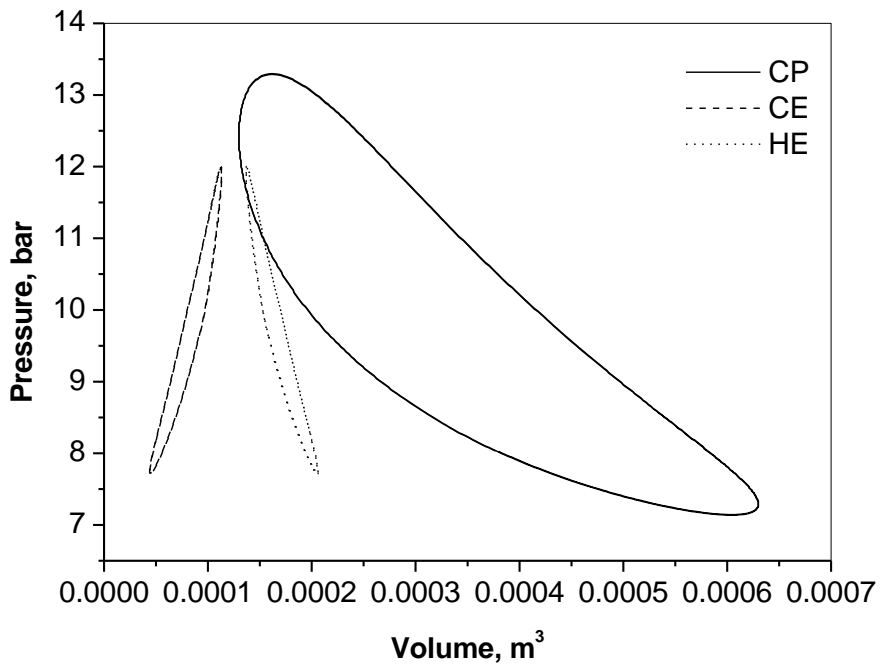

Figure 12. Compressor (CP), cold end (CE) and hot end (HE) work done in cyclic operation in case of DPTRs.

A Comparison among the BPTR, OPTR and DIPTR has been made based on an enlarged scale cold end work-done shown in Figure 13. BPTR shows no PV area, i.e. compression and expansion follow in the same path. But OPTR and DIPTR show their PV areas, i.e. having a cooling work. But DIPTR has performed the 
compression and expansion work within a shorter range compared to OPTRs with higher amplitude. That means DIPTR configuration provides more cooling capacity than that of the OPTR.

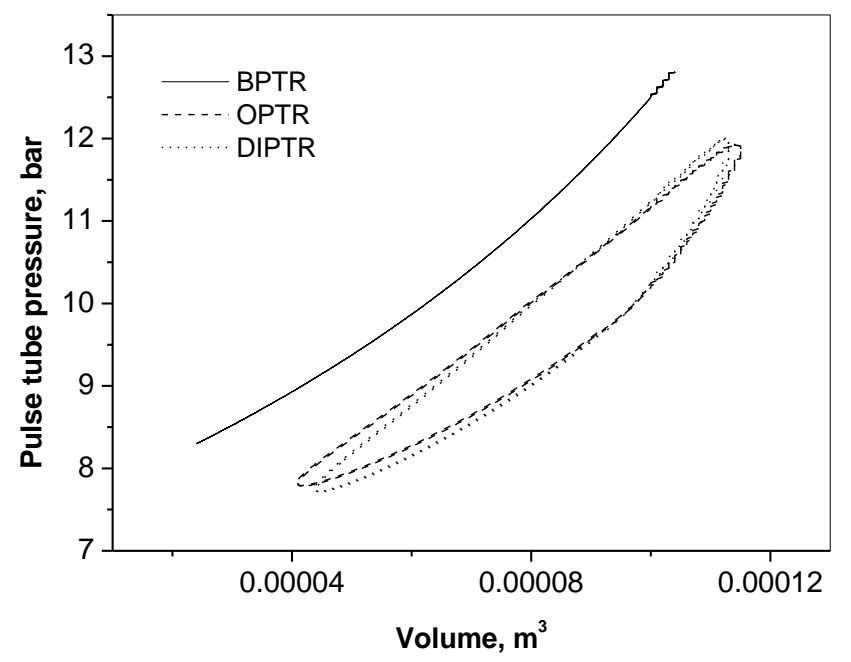

Figure 13. Cold end work done in case of BPTR, OPTR and DIPTR

\section{CONCLUSIONS}

To study the cooling effect at the cold-end of the Stirling type pulse tube refrigerator, a thermodynamic model has been proposed. This model has ability to predict pressure variations in the compressor, pulse tube and have been validated with in-house experimental results as a qualitative basis. It is observed from both the cases that though there have some quantitative difference between them, but a distinct time lag relationship among the pressures are observed in both the experimental results and model predicted results. However, model predicted results in compression and expansion are well validated with the experimental data. From the present model, it is understood the basic phenomenon which is responsible for the refrigeration effect in different types of pulse tube refrigerators (BPTR, OPTR and DIPTR). Time duration for expansion process is more than the compression process in case of OPTR and DIPTR, which leads to lower pressure during expansion and more cooling effect compared to the BPTR. A distinct comparison among three types of PTRs has been clearly shown for the cold end work done and DIPTR shows more cold-end PV-area compared to OPTR. Therefore, DIPTR shows always a better cooling capacity compared to OPTR and BPTR

\section{ACKNOWLEDGMENTS}

Authors wish to acknowledge the support from Prof. P. K. Das and Prof. S. Sarangi, I.I.T. Kharagpur and the team members of the PED Laboratory, Cryogenic Engineering Centre, IIT Kharagpur in doing experimental works to complete this research work.

\section{REFERENCES}

[1]Gifford, W. E., \& Longsworth, R. C. (1964). Pulse-Tube Refrigeration. Transactions of the ASME Journal of Engeenering for Industry, 86(3), 264-268.

[2]Mikulin, E. I., Tarasov, A. A., \& Shkrebyonock, M. P. (1984). Low-temperature expansion pulse tubes. In Advances in cryogenic engineering, 629-637.

[3]Shaowei, Z., Peiyi, W., \& Zhongqi, C. (1990). Double inlet pulse tube refrigerators: an important improvement. Cryogenics, 30(6), 514-520.

[4]Chopra, K., Sahni, V., \& Mishra, R. S. (2015). Energy, Exergy and Sustainability Analysis of Two-stage Vapour Compression Refrigeration System. Journal of Thermal Engineering, 1(4), 440-445.

[5]Arora, A., Dixit, M., \& Kaushik, S. (2016). Computation of Optimum Parameters of a Half Effect WaterLithium. Journal of Thermal Engineering, 2(2), 683-692.

[6]Radebaugh, R. (2000). Development of the Pulse Tube Refrigerator as an Efficient and Reliable Cryocooler. Institute of Refrigeration, 1999.

[7]Liang, J., Ravex, A., \& Rolland, P. (1996). Study on pulse tube refrigeration Part 1: Thermodynamic nonsymmetry effect. Cryogenics, 36(2), 87-93. 
[8]Cha, J. S., Ghiaasiaan, S. M., Desai, P. V., Harvey, J. P., \& Kirkconnell, C. S. (2005). CFD simulation of multi-dimensional effects in an Inertance tube pulse tube refrigerator. Cryocoolers, 13, 285-292.

[9]Zhang, X. B., Zhang, K. H., Qiu, L. M., Gan, Z. H., Shen, X., \& Xiang, S. J. (2013). A pulse tube cryocooler with a cold reservoir. Cryogenics, 54, 30-36.

[10]Banjare, Y. P., Sahoo, R. K., \& Sarangi, S. K. (2009). CFD simulation of a Gifford-McMahon type pulse tube refrigerator. International Journal of Thermal Sciences, 48(12), 2280-2287.

[11]Chen, L., Zhang, Y., Luo, E., Li, T., \& Wei, X. (2010). CFD analysis of thermodynamic cycles in a pulse tube refrigerator. Cryogenics, 50(11-12), 743-749.

[12]Boroujerdi, A. A., Ashrafizadeh, A., \& Mousavi Naeenian, S. M. (2011). Numerical analysis of stirling type Pulse Tube Cryocoolers. Cryogenics, 51(9), 521-529.

[13]Antao, D. S., \& Farouk, B. (2013). Experimental and numerical investigations of an orifice type cryogenic pulse tube refrigerator. In Applied Thermal Engineering (Vol. 50, pp. 112-123).

[14]Dai, Q., Chen, Y., \& Yang, L. (2015). CFD investigation on characteristics of oscillating flow and heat transfer in 3D pulse tube. International Journal of Heat and Mass Transfer, 84, 401-408.

[15]Park, J., Ko, J., Cha, J., \& Jeong, S. (2016). Stirling-type pulse tube refrigerator (PTR) with cold compression: Cold compressor, colder expander. Cryogenics, 74, 66-72.

[16] Zhang, X. B., Qiu, L. M., Gan, Z. H., \& He, Y. L. (2007). CFD study of a simple orifice pulse tube cooler. Cryogenics, 47(5-6), 315-321.

[17]Neveu, P., \& Babo, C. (2000). Simplified model for pulse tube refrigeration. Cryogenics, 40(3), $191-201$.

[18]Roy, P. C. Some Theoretical and Experimental Studies on Pulse Tube Refrigeration System. MS Thesis, Department of Mechanical Engineering, I.I.T. Kharagpur, India, 2004.

[19]Qiu, L. M., Zhi, X. Q., Gan, Z. H., Zhang, X. B., \& Zhang, X. J. (2014). Function of gas parcels in the pulse tube. International Journal of Refrigeration, 38(1), 358-366. 
Please review the attached article. Review should be based on your expert analysis, good information on the subject of the article and the relevant literature. Please review to point out any deficiencies in the text and, if necessary, provide instruction on the amendments or modifications to the text.

\section{General data on paper}

Paper Number: JTEN-2016-128

Paper Title: Thermodynamic Modeling of a Pulse Tube Refrigeration System

Date: 23.08 .2016

\section{[1] Articles's content rating}

\section{Evaluation Criteria}

Rounding numbers 1 to 5 evaluate each of these evaluation criteria of the articles content where 5

means complete suitability for publication while 1 absolute grounds for refusal of the article.

$$
\text { Article content assessment criteria }
$$

1 The paper is written under ,Instructions for Authors

Evaulation

2 Paper title reflects the content and purpose of the research.

3 Summary includes information important for understanding the content of the $\begin{array}{llllll}1 & 2 & 3 & 4 & 5\end{array}$ paper.

4 The introduction clearly defines the purpose and objective of the $1 \quad 2 \quad 3 \quad 4 \quad 5$ work/research.

$5 \quad$ Worked out a review of previous research in the treated area.

6 The methodology is clearly defined.

$\begin{array}{lllll}1 & 2 & 3 & 4 & 5\end{array}$

$\begin{array}{lllll}1 & 2 & \mathbf{3} & 4 & 5\end{array}$

\begin{tabular}{lllllll}
7 & Showing results support the applied methodology and conclusions & 1 & 2 & $\mathbf{3}$ & 4 & 5 \\
\hline 8 & The conclusion is based and contributes to the discharge of treated problems. & 1 & 2 & $\mathbf{3}$ & 4 & 5
\end{tabular}

9 Article is a contribution by the theory / practice.

\section{[2] Articles's organization rating}

Rounding numbers 1 to 5 evaluate each of these evaluation criteria of the articles content where 5 means complete suitability for publication while 1 absolute grounds for refusal of the article.

\section{Article organization assessment criteria} Evaulation

1 The article is well organized and conforms to the "Instructions for authors".

2 The extent of the article is appropriate (up to 16 A4 pages).

3 Figures, tables and pictures are corresponding.

4 Terminology and measurement units are aligned with the metrology rules.

5 The references reflect the topicality of the article.

\begin{tabular}{lllll}
1 & 2 & 3 & 4 & 5 \\
\hline & 2 & 3 & 4 & 5
\end{tabular}

6 References are cited as directed by (Harvard system).

7 The article is written in standard language, relevant and interesting

\begin{tabular}{lllll}
\multicolumn{5}{c}{ Evaulation } \\
1 & 2 & $\mathbf{3}$ & 4 & 5 \\
1 & 2 & $\mathbf{3}$ & 4 & 5 \\
1 & $\mathbf{2}$ & 3 & 4 & 5 \\
1 & 2 & $\mathbf{3}$ & 4 & 5 \\
1 & 2 & $\mathbf{3}$ & 4 & 5 \\
1 & 2 & $\mathbf{3}$ & 4 & 5 \\
1 & 2 & $\mathbf{3}$ & 4 & 5
\end{tabular}

\section{[3] Reviewer's recommendation}

Mark with "X" one of the options. You state the article should:

\begin{tabular}{|l|l|c|}
\hline 1 & Publish as is & \\
\hline 2 & Conditionally accept with minor revisions (editor will check) & \\
\hline 3 & Conditionally accept with the necessary changes as recommended by reviewer & $\mathrm{X}$ \\
\hline 4 & The article should be thoroughly changed & \\
\hline 5 & Reject & \\
\hline
\end{tabular}

\section{[4] General remarks and recommendations of reviewer}

Reviewer Comments: Results and discussion part is too short in comparison to other sections. It should be longer. Validation data should be given in a Table and mentioned more. Introduction cannot be accepted with 7 references. This part should have 15-20 latest references on the subject at least. Last pragraphs of Introduction should not be in this section. They should be moved in a related section. Fig. 1 should not be in introduction part. This part is problematic now.

Reviewer Decision: Major Revision 


\section{[5] Data on reviewer}

Name and surname: Ahmet Selim Dalkilic

Signature:

Place and date: 10.11.2016 
Please review the attached article. Review should be based on your expert analysis, good information on the subject of the article and the relevant literature. Please review to point out any deficiencies in the text and, if necessary, provide instruction on the amendments or modifications to the text.

\section{General data on paper}

Paper Number: JTEN-2016-128

Paper Title: Thermodynamic Modeling of a Pulse Tube Refrigeration System

Date: 4.10 .2016

\section{[1] Articles's content rating}

\section{Evaluation Criteria}

Rounding numbers 1 to 5 evaluate each of these evaluation criteria of the articles content where 5

means complete suitability for publication while 1 absolute grounds for refusal of the article.

\section{Article content assessment criteria}

1 The paper is written under „Instructions for Authors

Evaulation

2 Paper title reflects the content and purpose of the research.

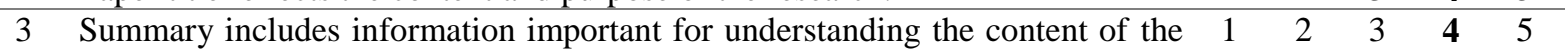
paper.

4 The introduction clearly defines the purpose and objective of the $1 \quad 2 \quad 3 \quad 4 \quad 5$ work/research.

5 Worked out a review of previous research in the treated area.

$\begin{array}{lllll}1 & 2 & 3 & 4 & 5\end{array}$

6 The methodology is clearly defined.

$\begin{array}{lllll}1 & 2 & 3 & \mathbf{4} & 5\end{array}$

$\begin{array}{lllllll}7 & \text { Showing results support the applied methodology and conclusions } & 1 & 2 & 3 & \mathbf{4} & 5\end{array}$

\begin{tabular}{lllllll}
\hline 8 & The conclusion is based and contributes to the discharge of treated problems. & 1 & 2 & 3 & 4 & $\mathbf{5}$ \\
\hline 9 & Article is a contribution by the theory / practice. & 1 & 2 & 3 & $\mathbf{4}$ & 5
\end{tabular}

\section{[2] Articles's organization rating}

Rounding numbers 1 to 5 evaluate each of these evaluation criteria of the articles content where 5 means complete suitability for publication while 1 absolute grounds for refusal of the article.

\section{Article organization assessment criteria} Evaulation

1 The article is well organized and conforms to the "Instructions for authors".

\begin{tabular}{lllll}
\multicolumn{5}{c}{ Evaulation } \\
1 & 2 & 3 & $\mathbf{4}$ & 5 \\
1 & 2 & 3 & $\mathbf{4}$ & 5 \\
1 & 2 & 3 & 4 & $\mathbf{5}$ \\
1 & 2 & 3 & 4 & $\mathbf{5}$ \\
1 & 2 & 3 & $\mathbf{4}$ & 5 \\
1 & 2 & 3 & $\mathbf{4}$ & 5 \\
1 & 2 & 3 & 4 & $\mathbf{5}$
\end{tabular}

\section{[3] Reviewer's recommendation}

Mark with "X" one of the options. You state the article should:

\begin{tabular}{|l|l|c|}
\hline 1 & Publish as is & \\
\hline 2 & Conditionally accept with minor revisions (editor will check) & X \\
\hline 3 & Conditionally accept with the necessary changes as recommended by reviewer & \\
\hline 4 & The article should be thoroughly changed & \\
\hline 5 & Reject & \\
\hline
\end{tabular}

[4] General remarks and recommendations of reviewer

Reviewer Comments: 1)The grammar and English has some common mistakes, some example are as: In Abstract, "Model predicted resultsare depicted to understand the...." In Introduction, "shown in Figure 1, the displacer is eliminated.The proper gas...." "with adiabatic compression and expansion in the pulse tube.The function of..." Revised the full manuscript in respect to such mistakes. 2) Add description of figure 4 3) Cite some latest and relevant research paper from the span 2010-2016

Reviewer Decision: Minor Revision 


\section{[5] Data on reviewer}

Name and surname: Parveen Sharma

Signature:

Place and date: 8.10.2016 
Please review the attached article. Review should be based on your expert analysis, good information on the subject of the article and the relevant literature. Please review to point out any deficiencies in the text and, if necessary, provide instruction on the amendments or modifications to the text.

\section{General data on paper}

Paper Number: JTEN-2016-128

Paper Title: Thermodynamic Modeling of a Pulse Tube Refrigeration System

Date: 4.10 .2016

\section{[1] Articles's content rating}

\section{Evaluation Criteria}

Rounding numbers 1 to 5 evaluate each of these evaluation criteria of the articles content where 5

means complete suitability for publication while 1 absolute grounds for refusal of the article.

$$
\text { Article content assessment criteria }
$$

1 The paper is written under ,Instructions for Authors

Evaulation

2 Paper title reflects the content and purpose of the research.

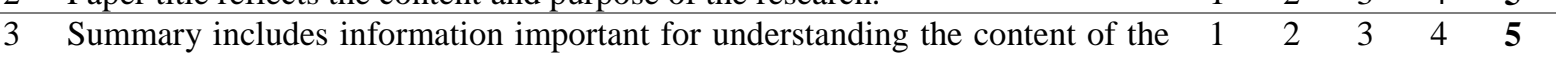
paper.

4 The introduction clearly defines the purpose and objective of the $1 \quad 2 \quad 3 \quad 4 \quad 5$ work/research.

5 Worked out a review of previous research in the treated area.

6 The methodology is clearly defined.

$\begin{array}{lllll}1 & 2 & 3 & 4 & 5\end{array}$

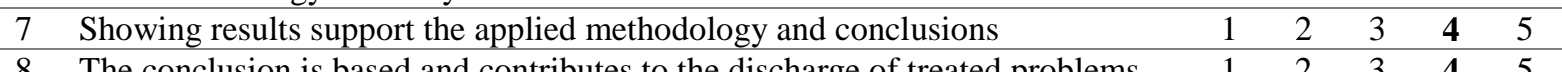

\begin{tabular}{lllllll}
\hline 8 & The conclusion is based and contributes to the discharge of treated problems. & 1 & 2 & 3 & $\mathbf{4}$ & 5 \\
\hline 9 & Article is a contribution by the theory / practice. & 1 & 2 & 3 & $\mathbf{4}$ & 5
\end{tabular}

\section{[2] Articles's organization rating}

Rounding numbers 1 to 5 evaluate each of these evaluation criteria of the articles content where 5 means complete suitability for publication while 1 absolute grounds for refusal of the article.

\section{Article organization assessment criteria} Evaulation

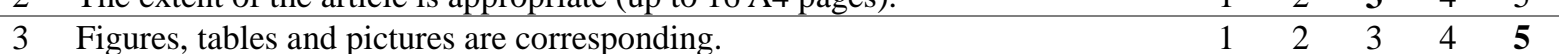

\begin{tabular}{lllllll}
\hline 4 & Terminology and measurement units are aligned with the metrology rules. & 1 & 2 & 3 & 4 & $\mathbf{5}$
\end{tabular}

$\begin{array}{lllllll}5 & \text { The references reflect the topicality of the article. } & 1 & 2 & \mathbf{3} & 4 & 5\end{array}$

\begin{tabular}{lllllll}
6 & References are cited as directed by (Harvard system). & 1 & 2 & 3 & 4 & 5 \\
\hline
\end{tabular}

7 The article is written in standard language, relevant and interesting

[3] Reviewer's recommendation

Mark with "X" one of the options. You state the article should:

\begin{tabular}{|l|l|c|}
\hline 1 & Publish as is & \\
\hline 2 & Conditionally accept with minor revisions (editor will check) & X \\
\hline 3 & Conditionally accept with the necessary changes as recommended by reviewer & \\
\hline 4 & The article should be thoroughly changed & \\
\hline 5 & Reject & \\
\hline
\end{tabular}

[4] General remarks and recommendations of reviewer

Reviewer Comments: There is a need to strengthen the abstract and conclusion. References must be increased. Reviewer Decision: Minor Revision

[5] Data on reviewer

Name and surname: Muhammad Zahid Saeed

Signature:

Place and date: 5.11 .2016 
Version (1)

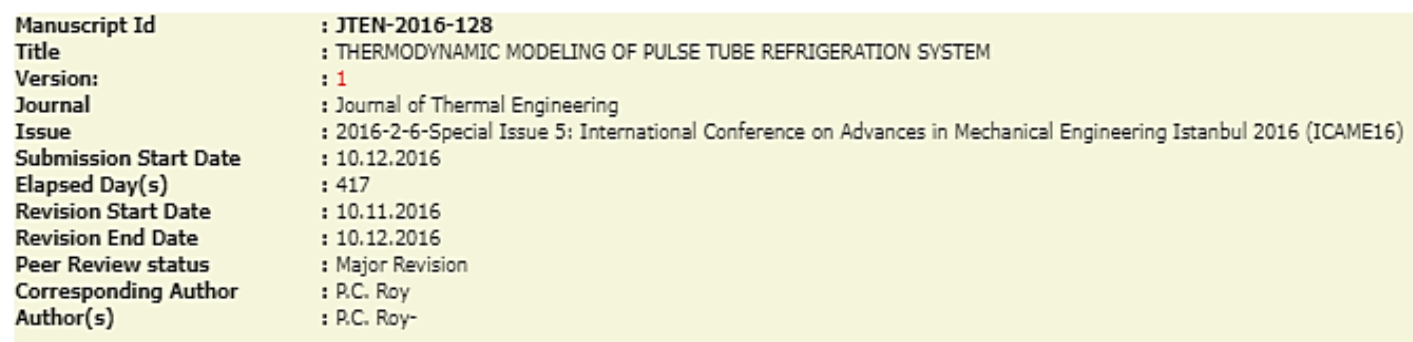

Manuscript Details

Manuscript Details

Manuscript Id

Title

Version:

Journal

Issue

First Submission Start Date: 14,6.2016(596)

Peer Review status

Corresponding Author

Author(s)

\section{: JTEN-2016-128}

$: 2$

:2018-4-1-January

: P.C. Roy
: Thermodynamic Modeling of a Pulse Tube Refrigeration System

: Journal of Thermal Engineering

: Published 12,12,2017(51)

: P.C. Roy- Balaram Kundu- 